\title{
INFRASTRUCTURES AND SCHEDULING METHOD FOR HOLONIC MANUFACTURING SYSTEMS
}

\author{
Nuno Silva, Carlos Ramos \\ Departamento de Engenharia Informática \\ Instituto Superior de Engenharia do Porto \\ Instituto Politécnico do Porto \\ Rua de S. Tomé, s/n \\ 4200 Porto - Portugal \\ Tel.: +35128340500 Fax: +3512821159 \\ e-mail: \{nsilva,csr\}@dei.isep.ipp.pt \\ http://www.dei.isep.ipp.pt/ \{nsilva, csr\}
}

\begin{abstract}
Manufacturing systems are changing its structure and organisation. Supply chain are evolving to more coupled organisations like virtual enterprises, though maintaining the single entities autonomy, adaptability and dynamism properties. Such organisations imply organisational and technological shift through agility, distribution, decentralisation, reactivity and flexibility. New organisational and technological paradigms are needed in order to reply to the modern manufacturing systems challenges. This paper proposes and justifies the Holonic Manufacturing System concept as the main organisational paradigm, presents infrastructures needed to assure system operation, security, coherence and coordination. Additionally, it is presented the scheduling sub-system along with a new scheduling method developed as case study.
\end{abstract}

Keywords: HMS, Multi-Agent, Architecture, Infrastructures and Scheduling.

\section{Introduction}

During the last years, enterprises felt the need to change approaches to market, reducing costs, time-to-market and product lifetime, at the same time increasing quality and environmental care. Hence, enterprise should be aware of concepts like concurrent engineering, virtual and federation enterprise, lean production, core business, etc.. These new organisation concepts lead to new approaches in production and therefore in Manufacturing Systems. Some different attitudes become central in modern manufacturing systems:

- Flexibility, ability to easily customise production;

- Reactivity, ability to automatically adapt production to reply to context modification;

- Distribution, different interrelated entities in the manufacturing system;

- Decentralisation, multiples entities producing the same element;

- Co-ordination, organise and harmonise production between different entities;

- Co-operation producing collectively by mutual requirement;

Furthermore, in a dynamic and unpredictable context, a broad concept has been developed: the Agile Manufacturing (Agility Forum, 97). This concept claims the manufacturing entity can no longer predefine its behaviour, its target market, product or services during it entire life cycle. The agile enterprise is an organisation that continually perceive environment and re-organise itself in order to satisfy momentary market demands, co-operating and competing in conjunction with similar entities. In this sense, the set of entities related to satisfy a specific market demand are no longer limited by its individual constraints, but complements them self forming a more responsive entity. Employing the Agile Manufacturing, the Virtual Enterprise or other related concepts (Silva, 98), main manufacturing operations become a distributed problem from the physical and from the logic point of view, thus MultiAgent Systems (MAS) and Holonic Manufacturing Systems (HMS) concepts are consider to be adopted.

In section 2, the Multi-Agent System and Holonic Manufacturing System paradigms are presented. Comparing their properties help explain why they were chosen as base paradigms for our project. In section 3, the Dynamic Scheduling Manufacturing System Architecture is presented and its entities and relations are described. In section 4, technological requirements are outlined in order to justify and present four holonic services infrastructures. In section 5 , the scheduling method is described. In section 6 , conclusions about 
already done work, and some remarks about questions not yet addressed are discussed.

\section{Multi-Agent Systems and Holonic Manufacturing Systems}

Until few years ago, CIM concept was considered satisfactory enough treating manufacturing enterprise requirements. However, taking in to account a new set of organisational and economic concepts, it becomes clear that the centralised CIM approach is not the answer. On the contrary, as stated in (Solberg and Kashyap, 93; Agility Forum, 97), these new concepts suggest autonomy, distribution, and flexibility, while stressing the need for co-operation among production units.

\subsection{Multi-Agent Systems}

An Agent is a rational entity sensing and acting on its environment in order to achieve its objectives. Several properties related to Agent like Autonomy, Social Ability, Reactivity, Pro-activeness (Jennings and Wooldridge, 95) are primary needs in modern manufacturing systems. A set of agents, when related by co-ordination rules and mechanisms in order to achieve defined a goal, is said to be a Multi-Agent System (MAS). Thus, a MAS can be understood as a society (or community) of agents where any of the entities consume and produce services from the others. Although MultiAgent System paradigm satisfies most of the new manufacturing systems requirements, several questions remain. For example, it has been referred the need for co-operation. The MAS concept supply different solutions but none is conceptual defined and accepted: master-slave, reacting, voting, negotiation (Davis, 83), constraints propagation and speech acts. Another example relates to structural configuration, should it be hierarchical or heterarchical? Consequently, MAS concept is too undetermined to satisfy organisational requirements (Silva, 98), which imply design ambiguity and incoherence.

\subsection{Holonic Manufacturing Systems}

The Holonic paradigm arises from Herbert Simon and Arthur Koestler studies about biological society evolution and organisation. Simon observed that complex systems are hierarchical systems formed by intermediate stable forms. These forms allow system to be stable, reliable and evolutionary, while maintaining a goal oriented functionality due to its hierarchical structure. Simon concluded that non-hierarchical systems are unnatural and inefficient and should be avoided in complex systems. Later, analysing Simon theory and comparing it with its own observations, Koestler (Koestler, 67) noticed that each system and its intermediate forms do not exist as auto-sufficient and non-interactive elements. On the contrary, they are simultaneously a part and a whole. A part since each element is always part of a bigger system, thus sub ordinate to the whole (upper levels), and a whole in the sense that each element is formed by multiple parts, thus supra ordinate its parts (lower levels) (Bongaerts et al., 96). In order to designate these hybrid nature and behavioural entities, Koestler (1967) proposed the terms Holon" and Holarchy. "A holarchy is a hierarchy of self-regulating holons, in supra-ordination to their parts, in sub-ordination to the higher levels and in coordination with environment" (Koestler, 67).

Furthermore, the IMS - HMS group systematised a set of properties related to the manufacturing systems based on the holonic paradigm:

- The holonic manufacturing system entities are autonomous and co-operative;

- Holon has information about itself and the environment;

- Each holon is composed by other holons and thus each holon is a also a holarchy;

- Each holon can dynamically belong to multiple holarchies;

- The holarchy has fixed rules and directives, the canon (Tharumarajah et al., 96).

Each holon must refine the proposed tasks, co-operating with its lateral partners with whom must share a limited set of competencies, in order to achieve its own goals and thus concur to achieve the global goal, defined by the higher holarchies. In this sense, the Holonic paradigm is an organisational and architectural concept since it defines the entity structures and its conceptual behaviours. The properties and behaviours defined for the holonic concept fits the structure and the dynamic behaviour of the modern manufacturing system requests, since it forms highly complex but reactive structures, making no (or little) compromise with efficiency.

However, the properties previously mentioned require implementation technology, which can be supplied by the MAS concept. Consequently, MAS is perceived as a technological concept, supplying mechanisms and development facilities, while HMS is seen as an organisational paradigm, specifying the architectural structure, co-ordination rules and behaviour. As a result, both concepts are mutual complementary and therefore appropriated as technological solution to implement modern manufacturing systems.

\section{A Dynamic Scheduling Manufacturing System Architecture}

As previously referred, a holonic manufacturing system is a holarchy composed by autonomous and co-operative entities, the holon, each one being another holarchy. The proposed architecture, represented in Figure 1, is based in (Sousa and Ramos, 96) and (Van Brussel et al., 97).

It is an holarchy composed of multiple high-level holons (e.g. Process Planning, Scheduling, Stock Management) responsible for the co-ordination of its sub-entities. Lower, the product, the resource and the task holons (among others). Considering the presented case study focus the scheduling activity, the figure emphasises the

${ }^{1}$ Holos (whole in greek) + on (a particle, like in neutron $)$ 
related holon, where are represented its interrelations between supra-ordinated and sub-ordinated entities.

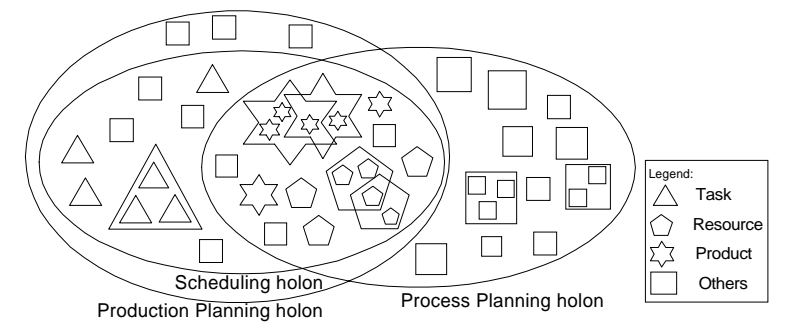

Figure 1 - Proposed architecture model (Silva, 98)

On one hand, the scheduling functionality relates to Production Planning the Scheduling holon sub ordinate to Production Planning holon, on the other hand, scheduling activity deals with different entities:

- The Product holon represents the type of product, not the product object itself. The complete set of Products represents the system production possibilities (the catalogue). The Product is responsible for product planning specification and production quality control (Van Brussel et al., 97). The Product can be composed by sub-Products, each one belonging to or be composed by others;

- The Task holon models a production task received in the system, each one constrained by products, resources, quantity and quality parameters. The Task holon is responsible for scheduling, control, execution and conflict avoidance between entities performing the task (Van Brussel et al., 97). The Task holon is potentially made of other Task holons, but contrary to Product, the Task can not belong to different Tasks;

- The Resource holon represents the production elements in the system Each Resource can be made of others (e.g. a working cell) and share it with other (e.g. a tool used by multiple machines). The Resource holon is responsible for the operation control and sub-resources co-ordination, and concurs to the scheduling process, defining agendas, capabilities and capacities.

However, these entities also concern with other activities like the Process Planning. Consequently, some entities are simultaneously part of two holarchies, and thus, must contain and co-ordinate the functionality of the different processes they belong to:

- Resource holon simultaneously engages in product process definition and scheduling activities. First, supplying capacity and functionality information needed to process planning, second, supplying time agenda and activity control information fundamental to dynamic scheduling;

- Product holon, concurrently contribute to the product process re-planning together with the Process Planning holon, and responsible by the quality control together with Production Planning holon.
Besides the prior description focus an intra-enterprise system, the same concept and structure can be expanded to inter-enterprises relations.

For example, as soon an enterprise perceives the market opportunity, starts to specify the product. Nevertheless, its technological skills can be insufficient to accomplish the entire product specification. Hence, the entity (modelled as a Product holon) engages in negotiation with other entities (modelled as Resource holons) in order to define product specifications. Meanwhile, each resource entity can itself be decomposed in different entities, thus forming a particular holarchy co-operating internally and externally, both with the Product and Resource holons.

In that sense, the presented architecture suits the holonic paradigm and the modern manufacturing concepts, described in section 1 .

\section{Holonic Services}

Holonic Manufacturing Systems architecture is more flexible and reactive than the traditional CIM architecture (Silva, 98, Sousa and Ramos, 97). However, being a dynamic and non-deterministic system, information coherence, security and availability are relevant topic that system architecture design should concern with. These questions concern the entire community and can not be achieved without all the entities co-operation and understanding about organisation. Nevertheless, it is convenient to supply complementary mechanisms to assist core entities ${ }^{2}$ to achieve such topics. Thus, a set of complementary entities was developed and its services combined into the system (Silva and Ramos, 99). Such entities, named Service holons are:

- Identification Service, responsible for identification, authentication and registration of holons in the holarchy. The service is assured by the co-operation of all the system Identification Service holons, based on security mechanisms achieved by a mutual authentication process;

- Information Service, responsible for the information management and co-ordination among multiples Information Service holons, while supplying information to core holons;

- Pooling Service, concerned with message delivery to holons temporarily unregistered in the holarchy. The service is requested by the holon that need to send messages to other;

- Routing Service is concerned with delivery and post-delivery messages. The holon that does not know where its conversation partner is located or it is not available in the moment, can request this service in order deliver messages. The routing service delivers the messages as soon as possible throughout the Pooling Service.

\footnotetext{
${ }^{2}$ Core entity is a system functional entity, responsible to accomplish specific tasks related to the main goal (section 3 specify different core entities).
} 
By the combination of these four services (Silva and Ramos, 99), it is possible to foster system coherence, information availability and some degree of security.

\section{Scheduling method and algorithm}

The scheduling system is triggered when a new production order arrives. A Task holon is created and will be responsible for the negotiation between resources, each one represented by an holon. The task holon requests all the resources that can perform each task operation, and therefore, each resource can receive several requests and each operation can be "negotiated" by several resources. An $n * m$ relation.

The original scheduling algorithm, developed for centralised manufacturing systems is described in (Ramos et al., 95). Its main priority is to schedule tasks considering due dates. Briefly, for each operation, one single resource is requested and each one calculates a time interval list considering the previous operation time interval list, respecting the time needed to perform the remaining operations until the due date. The time interval list calculated is passed to the resource requested for the next operation. Except the first resource, all the others must wait for the previous time interval list. This procedure is called the forward influence phase and finish when the resource is the last in the task plan (Figure 2). The time interval list found represent the last resource definitely availability to perform that operation.

Although this is the final list for the last operation, resources must influence previous resource intervals. Passing back its final list, resource informs the previous ones to readjust its previously suggested time intervals. This is called the backward influence phase (Figure 2) and finishes when the first resource readjusts its final list. Simultaneously with the backward influence phase, resource pass its list to the task holon, which, after receiving the list for all the operations, will initiate the time interval choosing process.

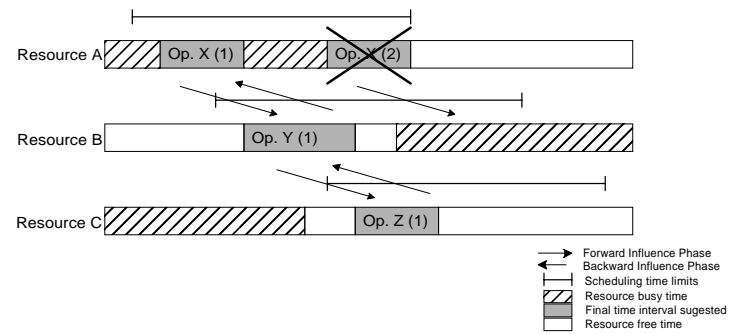

Figure 2 - Backward influence phase. Resource A reject $2^{\text {nd }}$ interval because there is no further correspondence (Silva, 98).

Some questions must be highlighted:

- Each operation is executable in one single resource. On the contrary, in the desired system, any operation can be "negotiated" by multiple resources. If the original algorithm logic is followed, for each time interval list received, the resource calculates an influenced list and passes it to the next resources. Therefore combination explosion easily occurs.

- The algorithm states that each resource must wait for the previous time interval list (back or forward). In requested system, is it reasonable to follow the rule or not? Should each resource wait and process all the previous lists, or can it discard some list depending on some options and heuristics? If it discards some intervals, there is no guarantee that the "better" solution (or just the solution) will be found. On the other hand, if discard does not happen then the time to achieve the solution can be prohibitive in a few operations and resources.

- The solution is calculated from a time interval list. It is very simple and can be optimised to local and global policies. However, in the developing system, each operation is possible in different resources, which adds a new dimension to the problem, thus affecting the choosing process efficiency and solution optimisation.

\section{1. $\quad$ Proposed Method}

To adapt the original algorithm to the holonic approach some modifications are proposed. Such modifications are based on assumption that each resource does not need to know what resource is suggesting the previous time interval. The method is divided in four different phases:

1 Forward influence phase;

2 Backward influence phase;

2.1 Time interval Classification;

3 Sequencing phase;

4 Selection phase.

In the forward influence phase process, the resource gathers the entire time spans in one single list, ignoring the resources identification. Further, each time span is compared with the resource free time. If the resource agenda allows the suggested interval to be used, the corresponding interval will be inserted in the time interval list that will be suggested to next resources. If the time span was no correspondence, it is discarded. This process is exemplified in Figure 3 . Resource $\mathbf{J}$ and $\mathrm{K}$, negotiating the execution of operation $\mathrm{X}$, receive the same time interval list. In the example, both "answer" to one time interval. Interval 3 is discarded since no resource has free usable time to execute the operation after it. Thus, to next resources, only two intervals are suggested. 


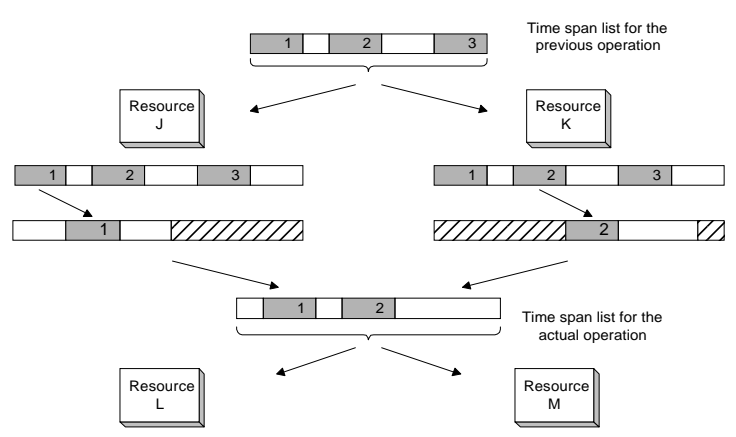

Figure 3 - Adapted forward influence phase.

In the backward influence phase, the process is repeated: the resource sends a single list to all the previous resources. The list represents the resource availability to perform the operation. In order to validate the previously suggested intervals, each resource compares them with the list received. Like in forward influence phase, if an interval is not corresponded, it is discarded and not further suggested. As soon as the resource defines the final time interval list, it must send the list to the Task holon. The example in Figure 4 represents the backward influence phase, and relates to the example in Figure 3. From the forward-suggested list, only interval 2 was further corresponded, which means Resource $\mathrm{J}$ will discard its interval. Thus, only interval 2 will be accepted and further utilised.

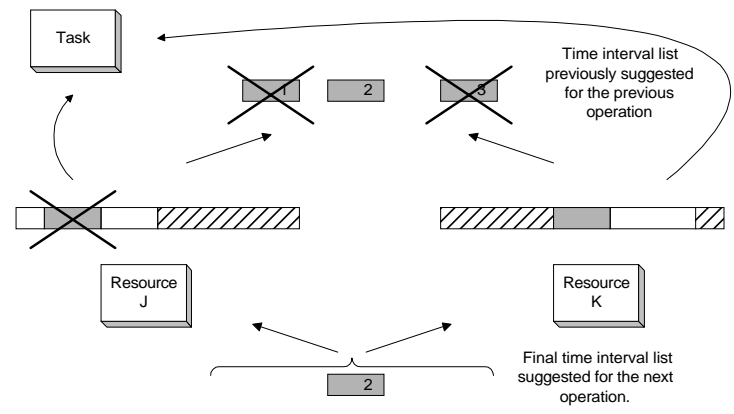

Figure 4 - Backward influence phase.

Since each resource receives several lists but sends only one, combination explosion is clearly avoided concerning message transfer. Consequently, each resource can wait for all the previous lists (forward or backward), that allows continuing find all the possible solutions. However, it is impossible to quantify the evolution of the number of time intervals treated during the scheduling process, since the intervals are highly dependable on the resources agendas, which are dynamic and unpredictable.

This question is very important in the choosing process, since the task must efficiently choose the better sequence of resource-interval. Notice that the problem already existed in the original algorithm, where some entity had to choose one of the different time intervals sequence suggested. The decentralised and negotiated approach adds one dimension to the problem: now there are different resources with different intervals for each operation, constituting more different possible sequences. In order to solve partially this problem, the method suggests the classification of time intervals based in some function, defined by the Task. The classification value can be considered a cost if market based approach is used or some other unit depending on the used approach. Two values are calculated:

- Local value, that represents the value the task needs to know about the execution in each resource. During the backward influence phase the time intervals for each operation and resource are completely defined. Hence, the resource can classify each time span considering a set of parameters previously defined by the Task holon;

- Global value represents the sum of all the local values in the resources sequence until the end of process.

In that sense, the time span must be complemented with information concerning that accumulated value (Figure 5).

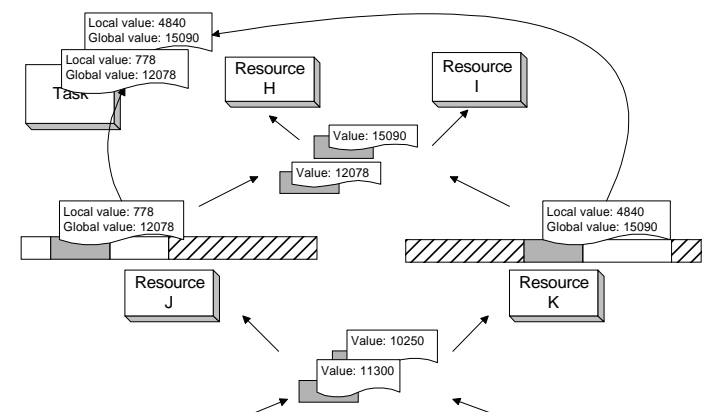

Figure 5 - Time interval classification with value propagation (Silva, 98).

Consequently, the Task holon knows for each suggested time interval the local value and the global value. Each time interval also contains information about suggesting resource and related operation.

The time intervals are stored based in its arrival, which is not adequate in the choosing phase. Since the time span classification value include both local and global parameters and it is presumably a good benchmark indicator, it is also suited to improve the choosing phase. Thus, the Task holon sorts the time intervals based on the global parameter in order to efficiently choose the solution. Substituting or complementing the classification method, some heuristics can be applied.

In case solution is not found, the task holon is notified although no reactive constraint relaxation mechanisms are automatically proposed. The user is responsible for the constraint relaxation mostly based on changes imposed to production process.

\section{Conclusions}

The described work is part of a case study developed in ISEP labs in order to test and validate MAS and HMS concepts when applied to modern manufacturing. In this study, focus is putted in dynamism, unpredictability and reliability. 
The HMS and MAS paradigms are briefly described and compared, which allows support the opinion both are compatible and moreover complementary, and not contradictory or opponent. The complementary services are briefly described. Some tests and simulations were done using the developed system, where the Service holons take an important role, guarantying security, mediating information exchange and facilitating negotiation. Although the results are not conclusive, it is now clear that the proposed approach based in complementary services is valid, adequate and satisfactory.

The dynamic scheduling architecture and its central components are described. Due to different conceptual requirements, the initial scheduling method suffers some changes in order to address modern manufacturing properties mainly distribution, decentralisation and unpredictability. Primary change make use of constraint propagation approach, which seems to be conceptually appropriated, but exhibit some pitfalls concerning autonomy and performance, i.e. the entity is autonomous in order to specify different operation parameters, but doing so, constraint propagation easily results in combination explosion, affecting indubitably the system performance. Time interval classification is another essential modification since it results in clear improvements in choosing phase.

Yet, some issues are not completely analysed or implemented:

- Concurrent operations are very common in product plans, though the proposed method comport several limitations. In the original method this was not a problem, since there is a previous phase denominated Behaviour Generation that defines the plan operation sequence and parallelism. In the holonic approach this is not totally possible since the operation length is not globally predefined in plan, but depends on particular resource. Since the resource set negotiating the operation is very dynamic during the system life cycle, it is not possible to specify operation length thus causing a contradiction between resource autonomy and system performance;

- Heuristics used in choosing process. Although heuristics strongly depends on particular implementation and utilisation, some studies should be done to validate method application;

- Application of constraint relaxation mechanisms must be analysed and adapted to the method;

- Due to distribution, time-out problems easily occur in resources conversations during time span propagation. Although this can be considered a control issue, the related problems affect considerable the scheduling method since in inter-enterprises negotiation the communication technology is commonly unreliable which decreases system performance.

Others case studies are being implemented namely related to electronic commerce. Although the process looks distinct there exist great similarities: the process is triggered by the user request, a new order is created and may decomposes it self in different others, which engages in negotiation to get the better product with better price, considering the best payment taxes, etc..

\section{REFERENCES}

Agility Forum, 97; http://www.agilityforum.com; 1997. Bongaerts et al., 96; L. Bongaerts, J. Wyns, J. Detand, H. Van Brussel, P. Valckenaers; Identification of Manufacturing Holons; Pre-Proceedings of the European Workshop on Agent Oriented Systems in Manufacturing; Berlin, Germany, 1996.

Davis, 83; Negotiation as a metaphor for Distributed Problem Solving; Davis R., Smith R. G.; Artificial Intelligence, vol. 20, no.1; 1983.

Jennings and Wooldridge, 95; Nick Jennings, Michael Wooldridge; Intelligent Agents: Theory and Practice; The Knowledge Engineering Review, vol. 10, pp. 115-152.

Koestler, 67; A. Koestler; The Ghost in the Machine; Hutchinson \& Co, London, 1967.

Ramos et al., 95; C. Ramos, Ana Almeida and Zita Vale, Scheduling Manufacturing Tasks considering Due Dates: a new method based on Behaviors and Agendas, Int. Conf. on Industrial and Engineering Applications of Artificial Intelligence and Expert Systems, Melbourne, Australia, 1995.

Silva, 98; Nuno Silva; Sistemas Holónicos de Produção - Especificação e Implementação; Dissertação de Mestrado; Faculdade de Engenharia da Universidade do Porto; September 1998.

Silva et al., 98; Nuno Silva, Paulo Sousa and Carlos Ramos; Proposal for a Dynamic Scheduling Architecture and Algorithm using an Holonic Approach; $4^{\text {th }}$ IFAC Workshop in Intelligent Manufacturing Systems 98; Gramado, Rio Grande do Sul, Brasil; November 1998.

Silva and Ramos, 99; Nuno Silva and Carlos Ramos; Holonic Dynamic Scheduling Architecture and Services; International Conference on Enterprise and Information Systems 99; Setúbal, Portugal; March 1999.

Solberg and Kashyap, 93; J. Solberg, R. Kashyap; Research in Intelligent Manufacturing Systems; Proceedings of the IEEE, vol. 81, n. 1, January 1993.

Sousa and Ramos, 96; P. Sousa, C. Ramos; Scheduling Orders in Manufacturing Systems using a Holonic approach; European Workshop on Agent-Oriented Systems in Manufacturing; Berlim, Germany; September 1996.

Sousa and Ramos, 97; P. Sousa, C. Ramos; A Distributed Architecture and Negotiation Protocol for Scheduling in Manufacturing Systems; ASI'98, Budapest. 
Tharumarajah et al., 96; A. Tharumarajah, A. J. Welles e L. Nemes; Comparison of the bionic, fractal and holonic manufacturing system concepts; International Journal of Computer Integrated Manufacturing, 1996, vol. 9, n 3, 217226.

Van Brussel et al., 97; Hendrik Van Brussel, Jo Wyns, Paul Valckenaers, Luc Bongaerts, Patrick Peeters; Reference Architecture for Holonic Manufacturing Systems: PROSA; Accepted for publication in Computers In Industry, special issue on intelligent manufacturing systems; 1997. 